\title{
Symmetry and degeneracy in microstructured optical fibers
}

\author{
M. J. Steel \\ Department of Applied Physics, Columbia University, 500 West 120th Street, New York, New York 10027, and \\ School of Physics, University of Sydney, Sydney, NSW 2006, Australia \\ T. P. White, C. Martijn de Sterke, and R. C. McPhedran \\ School of Physics, University of Sydney, Sydney, NSW 2006, Auistralia \\ L. C. Botten \\ School of Mathematical Sciences, University of Technology, Sydney, NSW 2008, Auistralia \\ Received December 4, 2000 \\ The symmetry of an optical waveguide determines its modal degeneracies. A fiber with rotational symmetry \\ of order higher than 2 has modes that either are nondegenerate and support the complete fiber symmetry or \\ are twofold degenerate pairs of lower symmetry. The latter case applies to the fundamental modes of perfect \\ microstructured optical fibers, guaranteeing that such fibers are not birefringent. We explore two numeri- \\ cal methods and demonstrate their agreement with these symmetry constraints. $\mathbb{O} 2001$ Optical Society of \\ America \\ OCIS codes: $\quad 060.2280,060.2420$.
}

Microstructured optical fibers (MF's), silica fibers with multiple air holes arranged around the core, are currently in vogue, mainly because of their unique dispersive properties. Fibers with one or two rings of large air holes, which behave like very large $\Delta n$ step-index fibers, allow solitons in the visible ${ }^{1}$ and low-power supercontinuum generation. ${ }^{2}$ With many more holes, typically arranged in a hexagonal lattice, photonic crystal fibers are obtained.

A scalar description of MF usually suffices if one wishes to obtain important properties such as group-velocity dispersion and effective nonlinearity. It has been recognized, however, that for some problems, such as bandgap guiding and large air-hole fibers, and accurate dispersion calculations, a complete vectorial model is required. ${ }^{3-6}$ The question then arises as to the existence of birefringence in these structures. Indeed, birefringence between the fundamental modes has been observed frequently. ${ }^{2,7-9}$

Theoretical discussion of MF birefringence has thus far been based almost entirely on numerical calculations of the modal spectrum, particularly of the pair of essentially uniformly polarized fundamental modes-the analogs in MF of the step-index fiber $\mathrm{HE}_{11}$ mode. Some authors (see, e.g., Ref. 5) have explicitly stated that these modes have different propagation constants, i.e., that they are nondegenerate. Others have referred to them as constituting a "doublet" but without defining this term rigorously. ${ }^{4,6}$ From the viewpoint of vector perturbation theory, splitting does not seem unreasonable for a hexagonal structure; for one polarization the field lines directly intersect an air hole, whereas for the other they pass between the holes (see Fig. 3, below), leading to different boundary conditions at the interfaces. ${ }^{5}$ It might seem unlikely that the fields should adjust themselves so that this difference does not lead to birefringent splitting.

0146-9592/01/080488-03\$15.00/0
Nonetheless, here we show that hexagonal photonic crystal fibers are not birefringent, and thus the fields adjust themselves in precisely the way described above. More generally, the modes of any waveguide with rotational symmetry of order $m>2$ either exhibit the full waveguide symmetry and are nondegenerate or occur in degenerate pairs that support this symmetry only in combination. Thus a mode with a preferred direction must be one of a pair of degenerate modes. In light of this result, we note that, although theoretical reports of birefringence exist, we are unaware of any papers in which the splitting has been quantified, nor do experimental observations of birefringence contradict our work here-small imperfections that break the rotational symmetry are clearly present in many images of fiber cross sections. Intentional symmetry breaking in photonic crystal fibers, of course, does lead to birefringence. ${ }^{9,10}$

As shown by McIsaac, ${ }^{11}$ the present problem, like all symmetry matters in physics, is naturally expressed in the language of group representation theory. Briefly, the symmetry operations of the index profile form an abstract group. A fiber with rotational symmetry of order $m$ maps to the point group $C_{m}$, whereas if there is also a line of reflection the appropriate group (of order $2 m$ ) is $C_{m v} .^{12}$ Group theory ${ }^{12}$ demands that the waveguide modes occur in finite sets with a common propagation constant. Each set forms a basis for an irreducible representation of the group. The dimension $d$ of the irreducible representation equals the mode degeneracy, and it is well known that $d \leq 2$ for all the relevant groups. ${ }^{11}$ McIsaac thus showed that the modes are either nondegenerate $(d=1)$, in which case they must support all the symmetry operations of the group, or come in degenerate pairs $(d=2)$, and the individual modes then support only a subgroup of the full symmetry. ${ }^{11}$

(C) 2001 Optical Society of America 
Although McIsaac's approach is powerful and general, a complete proof requires a detailed knowledge of group theory. As the finding is of broad interest, here we obtain the essential results by use of a simple argument. We need work only with the transverse electric field, $\mathbf{e}(\mathbf{x})=\left[E_{x}(x, y), E_{y}(x, y)\right]$, as both $E_{z}$ and the magnetic field may be obtained from $e$ by use of Maxwell's equations. We define the scalar product $\circ$ between two arbitrary electric fields:

$$
\mathbf{e}^{(1)}(\mathbf{x}) \circ \mathbf{e}^{(2)}(\mathbf{x})=\int \mathrm{d} \mathbf{x} \mathbf{e}^{(1)}(\mathbf{x}) \times\left[\mathbf{h}^{(2)}(\mathbf{x})\right]^{*},
$$

where $\mathbf{h}^{(2)}$ is the transverse magnetic field corresponding to $e^{(2)}$. Then any field $e^{\text {in }}(\mathbf{x})$ can be expanded in the complete basis set of modes as

$$
\mathrm{e}^{\text {in }}(\mathbf{x})=\sum_{\beta, \sigma}\left(\mathbf{e}^{\text {in }} \circ \mathbf{e}^{\beta, \sigma}\right) \mathbf{e}^{\beta, \sigma}(\mathbf{x}),
$$

where we use indices for both propagation constant $\beta$ and polarization $\sigma$ to account for degeneracies. The modes $\mathbf{e}^{\beta_{j}, \sigma_{j}}(\mathbf{x})$ are normalized such that $\mathrm{e}^{\beta_{i}, \sigma_{p}} \circ \mathrm{e}^{\beta_{j}, \sigma_{q}}=\delta_{i j} \delta_{p q}$. Now suppose the fiber has rotational symmetry of order $m$. We take any bound mode $e^{\gamma, \tau}$ and apply a $2 \pi / m$ rotation to the entire field to obtain a new state, $e^{\text {rot. }}$. Since the refractive index is unchanged, the rotated field must also be a mode, with the original propagation constant $\gamma$. If the field $e^{\gamma_{1} \tau}$ is itself $n$-fold symmetric, then $\left|\mathbf{e}^{\text {rot }} \circ \mathbf{e}^{\gamma, \tau}\right|=1$ and by orthogonality, $\mathbf{e}^{\text {rot }}$ and $\mathbf{e}^{\gamma, \tau}$ must be the same mode. Suppose, however, that $e^{\text {rot }}$ has some preferred direction. Again, $e^{\text {rot }}$ must be a mode with propagation constant $\gamma$. However, the angles of dominant polarization differ by $\theta=2 \pi / \mathrm{m}$, and thus $|\Gamma|=\left|\mathbf{e}^{\text {rot }} \circ \mathbf{e}^{\gamma, \tau}\right|<1$. Then $\mathbf{e}^{\text {rot }}-\Gamma \mathbf{e}^{\gamma, \tau}$ is a normalizable state, orthogonal to and degenerate with $e^{\gamma, \tau}$. Setting $m=6$ and recalling that the fundamental $\mathrm{HE}_{11}$ modes of a MF are approximately uniformly polarized ${ }^{3-5}$ and thus have a preferred direction, we find, contrary to earlier claims, that these modes must be degenerate, and MF's are thus not birefringent. As mentioned above, a property of the $C_{n}$ and $C_{n v}$ groups is that the degeneracy must be precisely twofold. Below we show that the first nondegenerate modes are quasi-TE and quasi-TM modes of sixfold symmetry, which are each split from a second degenerate pair of $\mathrm{HE}_{21}$ modes.

We illustrate our findings by use of the well-known plane-wave expansion method and a new multipole method developed by several of us. ${ }^{13}$ In the latter method, the field is expanded in cylindrical harmonics separately in each region, and the interface boundary conditions are enforced exactly. By construction, this method respects the full symmetry of any arrangement of holes, and so numerical birefringence is at the level of the computer's numerical precision.

In Fig. 1, we consider a fiber (background index $n=1.45$ ) with a ring of six air holes of diameter $5 \mu \mathrm{m}$, positioned $6.75 \mu \mathrm{m}$ from the center. ${ }^{7}$ Labeling the modes by their step-index analogs, we find that at $\lambda=1.55 \mu \mathrm{m}$ the lowest modes are $\mathrm{HE}_{11}$ ( $n_{\text {eff }}=1.444767275$, degenerate pair), quasi-TE $\left(n_{\text {eff }}=1.437035894\right), \quad \mathrm{HE}_{21} \quad\left(n_{\text {eff }}=1.436866701\right)$, degenerate pair), and quasi-TM $\mathrm{TM}_{0}\left(n_{\mathrm{eff}}=1.436773535\right)$; the values for $n_{\text {eff }}$ are the converged values of the multipole method. ${ }^{13}$ The convergence rate is illustrated in Fig. 1(a). For each mode $j$, we plot the difference $\Delta n_{\text {eff }}^{j}=n_{\text {eff }}^{j}\left(N_{\text {cyl }}\right)-n_{\text {eff }}^{j}\left(N_{\text {max }}\right)$, versus the total number of cylindrical harmonics per polarization, $N_{\text {cyl }}$, with $N_{\max }=133$. We emphasize that, for every value $N_{\text {cyl }}$, we find two HE states that are degenerate to the machine precision.

However, the plane-wave method, when it is used on a rectangular grid, introduces numerical birefringence, which splits the HE states. To be consistent with earlier theory, this splitting should decrease with increasing basis size. This expectation is confirmed in Fig. 1(b), which shows the convergence of several quantities with the number of plane waves per polarization, $N_{\mathrm{pw}}$. The upright and inverted triangles show the numerical splittings of the $\mathrm{HE}_{11}$ and $\mathrm{HE}_{21}$ states, respectively. We find degeneracy in the HE states to the order of $10^{-8}$, corresponding to differences near machine precision for $n_{\text {eff }}^{2}$. Note that for $2^{10}<N_{\mathrm{pw}}<2^{16}$ the $\mathrm{HE}$ curves flatten out, from which one might mistakenly predict splitting of the order of $10^{-6}$. With $N_{\mathrm{pw}}>2^{16}$, the correct trend to zero reappears, underlining the care that is required for these calculations. For the nondegenerate TE and TM states we show that quantities $\delta n^{\mathrm{TE}_{0}}=n_{\text {eff }}\left(\mathrm{TE}_{0}\right)-n_{\text {eff }}\left(\mathrm{HE}_{21}\right)$ and $\delta n^{\mathrm{TM}_{0}}=$ $-\left[n_{\text {eff }}\left(\mathrm{TM}_{0}\right)-n_{\mathrm{eff}}\left(\mathrm{HE}_{21}\right)\right]$. The TE and TM states converge to distinct values that differ from the $H_{21}$ index and from each other by amounts that are several orders of magnitude larger than the machine precision. Thus, with sufficient basis states, the plane-wave expansion method also correctly describes the degeneracies.

A more exotic fiber with sixfold rotational symmetry but no reflection axes is presented in Fig. 2. By our earlier arguments, this symmetry should still
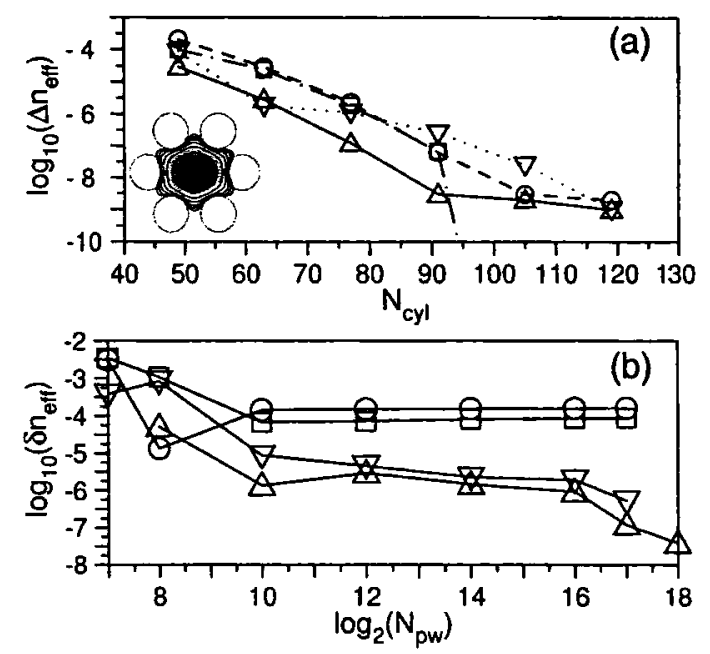

Fig. 1. (a) Convergence of $n_{\text {eff }}$ with $N_{\text {cyl }}$ for $\mathrm{He}_{11}(\Delta)$, $\mathrm{He}_{21}(\nabla), \mathrm{TE}_{0}(\mathrm{O})$, and $\mathrm{TM}_{0}(\square)$ modes. (b) Splittings with $N_{\mathrm{pw}}$ states per polarization: $\mid n_{\mathrm{eff}}\left(\mathrm{HE}_{11} x\right)-$ $n_{\mathrm{eff}}\left(\mathrm{HE}_{11} y\right)|(\Delta),| n_{\mathrm{eff}}\left(\mathrm{HE}_{21} x\right)-n_{\mathrm{eff}}\left(\mathrm{HE}_{21} y\right) \mid(\nabla), \delta n^{\mathrm{TE}_{0}}(\mathrm{O})$, and $\delta n^{\mathrm{TM}_{0}}(\square)$. 


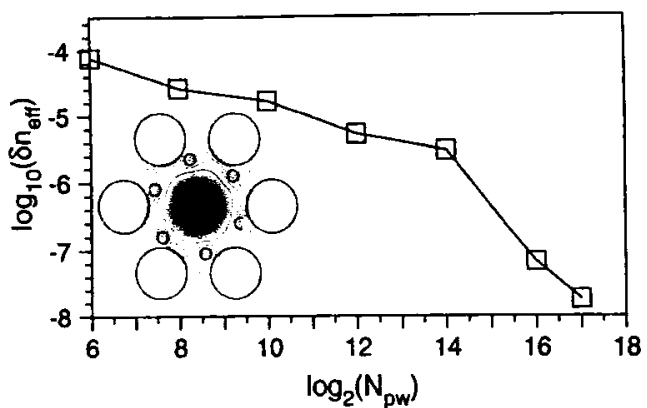

Fig. 2. Splitting of the $\mathrm{HE}_{11}$ mode for "satellite" fiber with the plane-wave method. The rightmost holes are located at $(1,0) \mu \mathrm{m}$ and $(0.575,0.658013) \mu \mathrm{m}$, with radii $2.3 \mu \mathrm{m}$ and $0.5 \mu \mathrm{m}$.

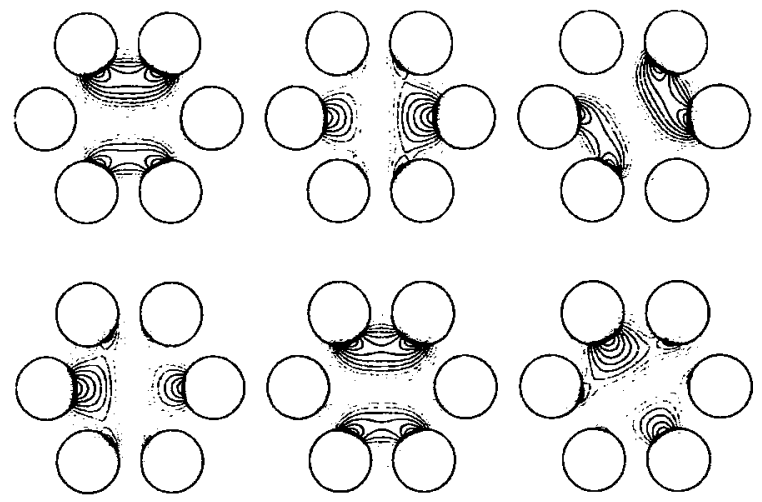

Fig. 3. $E_{z}$ (top row) and $H_{z}$ (bottom row) for orthogonal $\mathrm{HE}_{11}$ states $\mathrm{e}^{(1)}$ (column 1) and $\mathrm{e}^{(2)}$ (column 2). Column 3 is the superposition $\cos (2 \pi / 6) e^{(1)}+\sin (2 \pi / 6) e^{(2)}$.

produce degenerate fundamental modes. The multipole method predicts degeneracy with $n_{\text {eff }}=$ 1.44363507 at $\lambda=1.55 \mu \mathrm{m}$. In Fig. 2, we again plot the convergence of the numerical splitting from the plane-wave expansion method for the $\mathrm{HE}_{11}$ mode and ultimately find degeneracy to the level of $\delta n_{\text {eff }} \leq 2 \times 10^{-8}$ with $N_{\text {pw }} \approx 2^{17}$.

We illustrate our superposition argument in Fig. 3. The first two columns show $E_{z}$ (top) and $H_{z}$ (bottom) for the orthogonal $\mathrm{HE}_{11}$ states labeled $\mathbf{e}^{(1)}$ and $\mathbf{e}^{(2)}$, respectively, for the six-hole fiber. The third column shows the superposition state $\cos (2 \pi / 6) e^{(1)}+\sin (2 \pi / 6) e^{(2)}$, which is a perfect rotation of $e^{(1)}$, in accord with symmetry principles. Note that, although the $E_{z}$ and $H_{z}$ fields of orthogonal polarization are very similar, there are slight differences in the contour spacings, as required by the interface boundary conditions.

In conclusion, we have shown that symmetry imposes degeneracy constraints on fiber modes and demonstrated numerical results in accord with these constraints. In particular, we have established that microstructured optical fibers with sixfold rotational symmetry are not birefringent.

We thank Leon Poladian and Ian Bassett for an enjoyable discussion. M. J. Steel was funded by National Institute of Standards and Technology Advanced Technology Program Cooperative Agreement 70NANB8H4018. His e-mail address is mike@ rsoftinc.com.

\section{References}

1. W. J. Wadsworth, J. C. Knight, A. Ortigosa-Blanch, B. J. Mangan, and P. St. J. Russell, in Quantum Electronics and Laser Science Conference, 2000 OSA Digest Series (Optical Society of America, Washington, D.C., 2000), paper QFA2.

2. J. K. Ranka, R. S. Windeler, and A. J. Stentz, Opt. Lett. 25,25 (2000).

3. D. Mogilevtsev, T. A. Birks, and P. St. J. Russell, J. Lightwave Technol. 17, 2078 (1999).

4. J. Broeng, S. E. Barkou, T. Sondergaard, and A. Bjaklev, Opt. Lett. 25, 96 (2000)

5. A. Ferrando, E. Silvestre, J. J. Miret, P. Andrés, and M. V. Andrés, J. Opt. Soc. Am. B 17, 1333 (2000)

6. T. M. Monro, D. J. Richardson, N. G. R. Broderick, and P. J. Bennett, J. Lightwave Technol. 18, 50 (2000).

7. B. J. Eggleton, P. S. Westbrook, C. A. White, C. Kerbage, R. S. Windeler, and G. L. Burdge, J. Lightwave Technol. 18, $1084(2000)$.

8. J. C. Knight, J. Arriaga, T. A. Birks, A. OrtigosaBlanch, W. J. Wadsworth, and P. St. J. Russell, IEEE Photon. Technol. Lett. 12, 807 (2000).

9. A. Ortigosa-Blanch, J. C. Knight, W. J. Wadsworth, J. Arriaga, B. J. Mangan, T. A. Birks, and P. St. J. Russell, Opt. Lett. 25, 1325 (2000).

10. M. J. Steel and J. R. M. Osgood, Opt. Lett. 26, 229 (2001).

11. P. R. McIsaac, IEEE Trans. Microwave Theory Tech. MTT-23, 421 (1975).

12. M. Lax, Symmetry Principles in Solid State and Molecular Physics (Wiley, New York, 1974).

13. T. P. White, C. M. de Sterke, R. C. McPhedran, and L. C. Botten, are preparing a manuscript to be called "Multipole method for microstructured optical fibers." 